Tifft: If $18 \cdot 5$ is taken as the Cloud modulus the cluster type variables may become uncomfort. ably faint $\approx+0 \cdot 8=M_{V}$.

Blaauw: I doubt whether the available evidence on the absolute magnitude of the RR Lyrae in particular is strong enough to cause any concern about the different distance moduli derived from these objects in the Clouds and the supergiants.

Walraven: The galactic supergiants may be underestimated.

Arp: Determinations from globular clusters give $M_{V}=+0^{\mathrm{m}} \cdot 3$ and $M_{B}=+0^{\mathrm{m}} 5$ as the best values for the $R R$ Lyrae stars. This gives a reasonable value for the galactic centre distance fainter values for the RR Lyrae stars would give an even smaller distance than $8 \cdot 2 \mathrm{kpc}$.

Blaauw: Considering the probable errors there is no basis for concern.

Arp : A point of more uncertainty is that there are only a few RR Lyrae stars in the Clouds and they may be unrepresentative.

\title{
67. SPECTROSCOPIC WORK IN THE MAGELLANIC CLOUDS: NGC 330 IN THE SMC
}

\author{
M. W. Feast \\ Radcliffe Observatory
}

This paper gives the results of a spectroscopic study of individual stars in the SMC cluster NGC 330. Before this, however, it may be useful to review briefly the spectroscopic evidence bearing on the questions of reddening and chemical composition in the Magellanic Clouds-matters about which there has not been entire agreement in the past.

Earlier Radcliffe results (Feast, Thackeray, and Wesselink 1960) showed that for the early-type supergiants in any spectral class the least reddened stars in the Magellanic Clouds agreed well in colour with Johnson's (1958) intrinsic colours except that amongst the A type supergiants Feinstein's (1959) intrinsic colours were to be preferred. The Radcliffe results gave a clear indication of interstellar reddening in both Clouds, although the mean total absorption was small for the stars studied ( $\sim \frac{1}{3}$ magnitude). Various lines of evidence lead to the conclusion that these reddenings are real and not, for instance, due to errors in the spectral types or intrinsic colours.

(1) Reddening is found from $U B V$ photometry as well as from spectral types and $(B-V)$ colours, as Wesselink's (1962) recent work amply confirms.

(2) The possibility that the wide range in $(B-V)$ observed at a given spectral type amongst the Radcliffe stars might be due to a strong dependence of intrinsic colour on absolute magnitude, with essentially no reddening, has been investigated. However, the correlation which would then be expected within a given type between $(B-V)$ and magnitude is not found. An apparent exception to this result occurs amongst the LMC B0 stars. In this spectral class three stars with $V$ near 12.55 are systematically bluer than three with $V$ near $11 \mathrm{~m} 5$. However, the three faint stars are all in the region near HDE 271191, which is on the outskirts of the LMC where the mean absorption is known to be low.

(3) The LMC star with the greatest known reddening was studied chiefly because it lay in an apparent absorption lane. Furthermore, the reddening is sys- 
tematically higher for stars seen in LMC gaseous nebulae than for those outside nebulae. This may be taken to indicate not only the presence of absorption but also that some at least of it occurs within the Clouds themselves.

(4) Observations of distant galactic foreground stars indicate a total visual absorption of about $0 \mathrm{~m} 2$.

(5) Interstellar absorption lines due both to Cloud and galactic gas have been found in stars in both Clouds.

(6) Earlier conclusions that the SMC was essentially dust free, since background nebulae could be seen through it, have not been confirmed (Wesselink 1961). A normal (galactic) ratio of dust to gas appears possible from this work.

So far as problems of chemical composition are concerned, the Radcliffe spectroscopic work gave no indication of any general peculiarities either for supergiants or gaseous nebulae. In the case of SMC supergiants the recent Mount Stromlo work (Buscombe and Kennedy 1962) confirms these conclusions. It is important to realize also that the matter does not rest on the examination of spectra alone. Comparison of the assigned spectral classes with the measured $U B V$ colours is extremely valuable in this respect. Contrary to the conclusions of some other workers, there is good agreement between the three-colour work and the independently assigned spectral types in both Clouds (Feast 1961; Wesselink 1962).

Arp's (1958, 1959) important work on the colour-magnitude diagrams of clusters in the SMC led him to believe that the young stars in this system were metal poor. Whilst a different interpretation of these results appears possible (Feast 1960), it was clearly desirable to carry out further observational work on these clusters and therefore a spectroscopic investigation of the brightest stars in the SMC cluster NGC 330 was made.

NGC 330 is a rather crucial cluster in that Arp concludes that it consists of young stars of age similar to those in $h$ and $\chi$ Per, but with low metal content. Essentially the arguments are, firstly, the existence of a gap in the colour-magnitude diagram between the top of the evolved main sequence and the supergiant branch and, secondly, the distribution (Hess diagram) of supergiants in colour. However, the results presented earlier in this Symposium (this volume, paper 5) confirmed a previous suggestion (Feast 1960) that this gap does occur in young clusters in the Galaxy and also that the Hess diagram is rather sensitive to absolute magnitude for these bright supergiants.

Spectra at $86 \AA / \mathrm{mm}$ have been obtained for eight supergiants in NGC 330 with $(B-V)$ between $0 \mathrm{~m} 0$ and $+0 \mathrm{~m} 6$. Some of these stars have in addition been observed with the new Radcliffe $f / 1$ Schmidt camera (170 $\AA / \mathrm{mm}$ ) and integrated spectra of the cluster have been obtained with this camera. The integrated spectrum shows signs of compositeness but in the main the match for a type near B5 (not a supergiant) is probably satisfactory. The results for the individual stars are shown in Table 1. It should be pointed out that the table includes all four stars for which Arp's $(B-V)$ values are between $+0 m_{1} 1$ and +0 m. The results are at first sight very startling. Stars with the same spectral type differ in colour by over half a magnitude. 


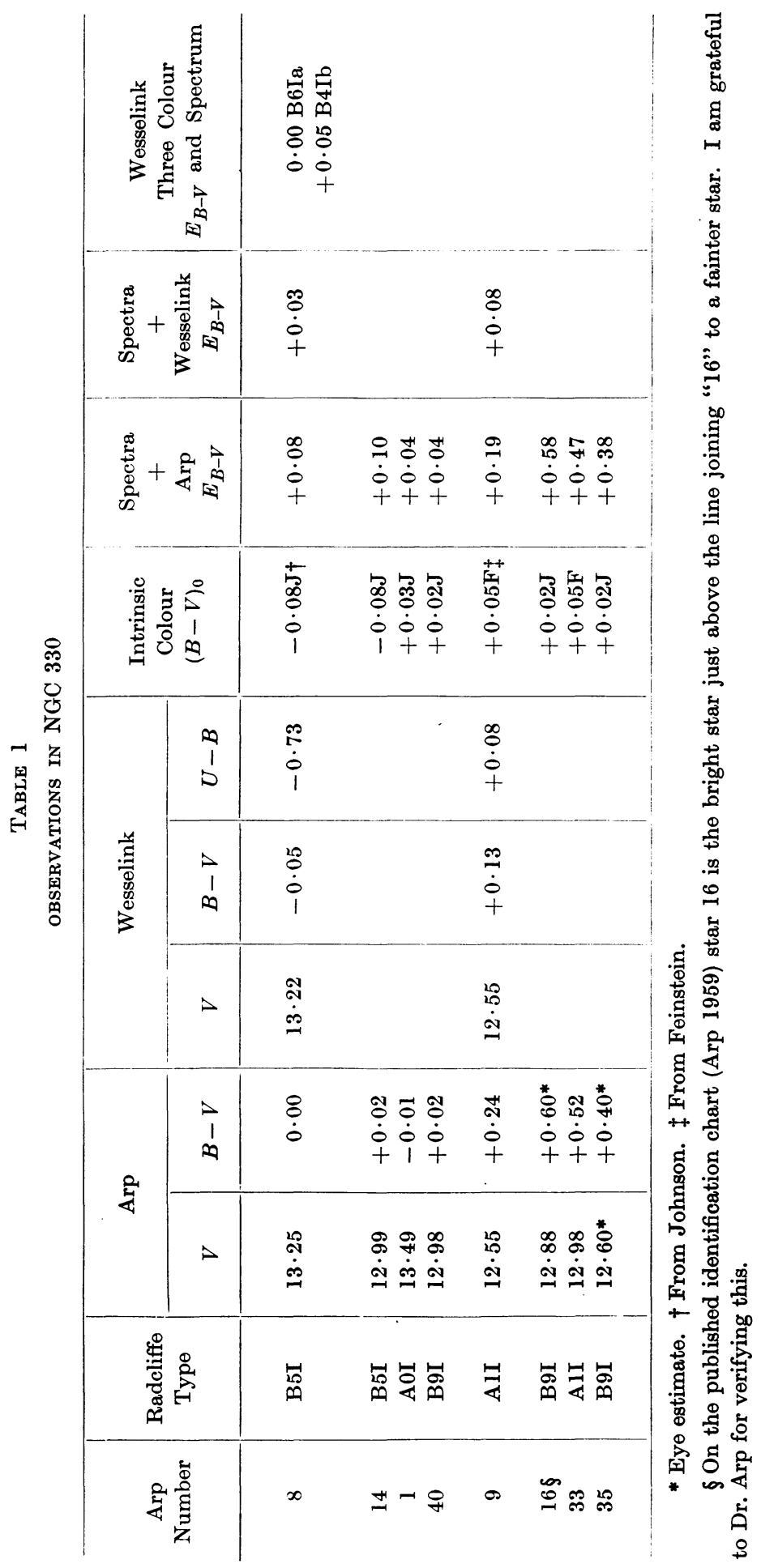


Two stars, numbers 8 and 9 , lie sufficiently far from the centre of the cluster that the photographic photometry may be checked photoelectrically. Dr. Wesselink has done this and has kindly allowed me to include his results in the table. His $(B-V)$ is bluer than Arp's in both cases, in one case $0 \mathrm{~m} l$ bluer. Since many of the other stars observed lie in a very densely populated area where even photographic measures must be very difficult, errors of at least 0 m 1 must probably be expected. In the case of star 8, Wesselink's $U B V$ photometry agrees beautifully with the spectral type. The $\mathrm{Q}$ method gives $\mathrm{B} 6$ if the star is Class Ia and B4 if it is of Class Ib and reddenings in the two cases of $0 \mathrm{~m}_{0}$ and $+0 \mathrm{~m} 05$. The spectrum itself yields B5 I and +0m03. For star 9 at type Al the data for the application of the $\mathbf{Q}$ method are not available but Wesselink's $U B V$ values fit well with the little published data at this type. The reddening obtained $(+0 \mathrm{~m} .08)$ agrees as well as can be expected with that of star 8 . The photographic $(B-V)$ would indicate a much higher reddening.

Considering possible uncertainties in the photographic $(B-V)$ 's the three stars 14, 1, and 40 have colours which may be expected for little reddened stars of these types. They indicate a mean reddening of +0 m06. All these results are therefore quite reasonable and do not give any indication of any abnormality in the cluster. However, the three stars at the bottom of the table have colours too red for their types by 0 m.5. If these stars were only slightly reddened we would expect spectral types in the late F's. An explanation in terms of interstellar absorption does not appear very likely. It is also very difficult to see how the effect could be explained as due to metal poorness in the cluster. The spectrum of a $B$ type supergiant is different from that of a late F star, not only in the absolute strengths of the metal lines but also of course in the general level of excitation. The stars observed in NGC 330 definitely appear to be high temperature stars (A or B). In any case metal weakness does not explain how two stars of the same assigned spectral type may differ by 0.5 in colour.

A possible explanation of this phenomenon appears if we consider what would happen if we put a typical young galactic cluster at the distance of the Magellanic Clouds and tried to measure its colour-magnitude diagram. The two clusters NGC 3293 and NGC 4755 (this volume, paper 5) may be taken as typical. They contain $B$ type supergiants plus one $M$ supergiant each. The main effect of removing these clusters to the Clouds is, of course, a violent reduction in scale (in NGC 4755 30" becomes 1".3). The main difficulty of obtaining a colour-magnitude diagram clearly becomes one of resolution. In the case of both NGC 4755 and NGC 3293 the M star would not be resolved from at least two other bright early-type stars as well as a number of fainter ones. Calculations indicate that the $M$ type stars will redden these clumps of stars without affecting the spectrum in the blue region. In the two cases, colours $0 \mathrm{~m} 21$ and $0 \mathrm{~m} 78$, too red for the types observed, will be found. It seems possible that such effects could occur in NGC 330. Whether such is, in fact, the explanation of the present observations may be tested when multicolour photometry becomes available. Spectroscopic investigations at long wavelengths and also possibly positional measurements for the stars on plates in different colours may help to solve this problem. 


\section{References}

ARP, H. C. (1958).-A.J. 63: 273-82 and 487-91.

ARP, H. C. (1959).-A.J. $64: 175-82$ and 254-8.

Buscombe, W., and Kennedy, P. M. (1962).-J.R.A.S. Canada 56 : 113-23.

Feast, M. W. (1960).-Observatory 80 : 104-6.

Feast, M. W. (1961).-Observatory 81 : 73-4.

Feast, M. W., Thackeray, A. D., and Wesselink, A. J. (1960).-M.N. 121 : 337-85.

Feinstein, A. (1959).-Zs. f. Ap. 47: 218-24.

Johnson, H. L. (1958).-Lowell Obs. Bull. 4: 37-46.

Wesselink, A. J. (1961).-M.N. 122 : 503-7.

Wesselink, A. J. (1962). - M.N. 124: 359-69.

\section{Discussion}

Buscombe: Have you radial velocities for individual stars in NGC 330?

Feast: I have not yet measured my plates for radial velocity.

Tifft: I must agree that photographic photometry in crowded regions is subject to background fluctuations which can be serious, especially as it affects colours.

Arp: Has Wesselink checked my local photoelectric sequence?

F'east: Wesselink has and agrees well with you.

Arp: Some preliminary results on the colour-magnitude diagram of NGC 1866 by Thackeray and myself show a diagram quite similar to that of NGC 458 in the SMC. It is not certain yet whether the differences - a generally slightly redder placement of the red giants - are due to a slightly different age - or represent an intrinsic difference between the two clusters.

Eggen: What certainty is there that the feature you show in the red giants of NGC 458 and 1866 is not caused by field stars?

Arp: In all the published work the control diagrams show the field to contribute negligibly.

Woolley: As far as I can remember without the diagram in front of me, there is nothing at all like this feature in NGC 1810, 1818, or 2004, all blue clusters in the LMC.

Gascoigne: We have colour-magnitude diagrams for a good many young clusters in the LMC and none show stars of the intermediate colours that Arp finds in NGC 330, 458, and 1866.

Westerlund: So far I have found no cluster in the LMC like NGC 458 but I will show three clusters in the SMC similar to NGC 458 in my paper later this afternoon.

Thackeray: Participants may recall that the colour-magnitude array of the rich galactic cluster NGC 6067 resembles somewhat that of NGC 1866 with a rising red branch, but that there was one directly observed composite star of intermediate colour which had to be split in the colour-magnitude array into a blue and red star.

Rodgers: I have an integrated spectrum of NGC 458: it is A5.

Thackeray: I never published a classification (of NGC 1866) but it was quoted as A3.

de Vaucouleurs: What is the absolute magnitude of the cepheid gap in the diagram Arp drew on the board?

Arp: The absolute magnitude of the brightest stars in NGC 330 is about $M_{V}=-6 \cdot 5$ mag. 